Title:

Depletion of the Chimeric Drug Rituximab from Biological Samples

Authors:

Benita K. Book ${ }^{\mathrm{a} 1}$

Mark D. Pescovitz ${ }^{\mathrm{a}}$

Lili Guo ${ }^{\text {b }}$

Eric A. Wiebke ${ }^{a}$

${ }^{a}$ Indiana University School of Medicine

Department of Surgery

635 Barnhill Drive

Indianapolis, IN 46202

U.S.A.

${ }^{\mathrm{b}}$ Peptide Core Facility

Indiana University School of Medicine

1345 West $16^{\text {th }}$ Street, Room 320

Indianapolis, IN 46202

U.S.A.

${ }^{1}$ Corresponding author:

Benita K. Book

Permanent address:

1724 E. Maynard Drive

Indianapolis, IN 46227

U.S.A.

Email: bbook@iupui.edu

Anti-lymphocyte antibodies (ALA) are used extensively in organ transplantation as immunosuppressive agents. These antibodies may be of non-human species, human, humanized, chimeric, or a combination of these. Rituximab, sold as Rituxan ${ }^{\mathrm{TM}}$, is a humanized chimeric monoclonal antibody that is approximately $25 \%$ murine (anti-CD20 antibody 2B8) and 75\% human antibody. Specifically, the Fc portion of rituximab is human IgG1 that can bind to human complement and initiate antibody dependent cellular cytotoxicity (ADCC) as well as complement dependent cytotoxicity (CDC). Rituximab was initially developed to treat $\mathrm{B}$ cell lymphoma via its ability to bind to the CD20 molecule on B cells. Several different mechanisms of action have been proposed and, in all, the end result is B cell depletion, both neoplastic and normal. Recently, rituximab has

This is the author's manuscript of the article published in final edited form as:

Book, B. K., Pescovitz, M. D., Guo, L., \& Wiebke, E. A. (2017). Depletion of the Chimeric Drug Rituximab from Biological Samples. Human Immunology. https://doi.org/10.1016/j.humimm.2017.05.003 
been included as part of pre-transplant conditioning regimens among allosensitized patients awaiting renal transplantation. Post-transplant, rituximab has been used to prevent and/or treat allograft rejection (both acute and chronic) and to facilitate $\mathrm{ABO}$ mismatched kidney transplants (1). When used in these clinical situations, the presence of rituximab in patient sera may interfere with transplant immunologic monitoring.

Preformed alloantibodies (predominantly HLA specific) in a sensitized individual react with antigens expressed on the vascular endothelial cells of organ allografts. Such antibodies can activate the classical complement pathway resulting in rapid necrosis of the transplanted tissue. Prior sensitization of recipients to donor alloantigens occurs by pathways including blood transfusion, pregnancies and previous allografts. The performance of a crossmatch immediately before the transplant is still performed as a measure to prevent hyperacute or accelerated allograft rejection. As well, crossmatch monitoring may be performed after transplant. Thus, the persistence of rituximab in patient serum/plasma can negatively impact crossmatch and alloantibody analysis. Since rituximab is cytotoxic in the presence of complement, sera that contain rituximab may result in a positive cytotoxic crossmatch assay, a result that would be mistakenly attributed to donor directed HLA alloantibodies. Additionally, the human portion of the chimeric monoclonal antibody provides a target for the anti-human Ig fluorochromes, thereby resulting in a positive flow cytometric crossmatch. Clearly, it is challenging to perform transplant crossmatch analysis when exogenous anti-lymphocyte antibodies such as rituximab are present. To overcome this issue, several methods can be applied. One method uses pronase, a proteolytic enzyme that removes CD20 from the surface of B cells by Fc receptor digestion (CD20 is a member of the Fc receptor family of proteins) (2). After CD20 is removed, rituximab typically no longer binds B cells, thereby making it possible to clearly detect class I and II Major Histocompatibility (MHC) antibodies using B cell targets. An alternative to Fc receptor digestion is to adsorb rituximab from the serum onto microparticles coated with polyclonal anti-mouse antibody (3).

Herein, we modify the conjugated bead assay described above. Instead of an anti-mouse antibody, a small fragment of the extracellular CD20 structure (residues 163 - 187) that 
specifically binds rituximab is conjugated to a microparticle suspension. Conjugating the CD20 peptide to biotin facilitates removal of rituximab, thereby permitting binding to streptavidin-conjugated magnetic particles.

Methods and Materials:

Peptide preparation:

Peptide was prepared at the Peptide Core Facility, Indiana University School of Medicine, Indianapolis, IN.

The synthesis of cyclic peptide [NIYNCEPANPSEKNSPSTQYCYSIQ-K(PEG 2 -Biotin)G, with S-S bond] was started on Fmoc-Gly-Wang resin (Midwest Bio-tech, Fishers, IN) using Fmoc chemistry. After the Lys(Mtt ${ }^{26}$ was coupled, the peptide-resin bearing the Mtt-protected lysine was treated with TFA/TIS/DCM (1:5:94) to remove Mtt group. The N-Biotinyl-NH-(PEG) $)_{2}$ COOH.DIPEA (Nova Biochem, Billerica, MA) was conjugated to the $\varepsilon$-amino group of lysine. The continuing synthesis of the peptide was carried out on an ABI 433A peptide synthesizer (Applied Biosystems Inc. Foster City, CA), using Fmoc chemistry and trityl-protected Cys. The cleavage of the peptide from resin and side-chain protecting group removal was accomplished using trifluoroacetic acid cocktail solution and the crude peptide was precipitated with ether and lyophilized. The disulfide bond formation of the peptide was carried out in $10 \%$ of dimethyl sulfloxide in phosphate buffered saline (PBS) at $\mathrm{pH}$ 7.4. The final peptide was purified by $\mathrm{C} 18$ preparative reverse-phase HPLC and was characterized by analytical HPLC and molecular weight analysis using mass spectrometer.

\section{Conjugation to Beads:}

The purified peptide was reconstituted with $0.1 \%$ bovine serum albumen (BSA). Twentyfive nMoles of solubilized peptide were added to 16.75 X $10^{6}$ washed Dynal M-280 super-paramagnetic polystyrene beads (Invitrogen, Carlsbad, CA). The peptide and beads, precoated with streptavidin covalently attached to the hydrophobic surface, were incubated for 30 minutes at room temperature in a 12 X 75 polystyrene tube. No difference in absorption was noted with twice or half as much peptide for the same amount of beads, or when the incubation time was changed in time intervals ranging from 
$15,30,40,60,75$ minutes to 4 hours. Excess peptide solution was removed by adhering the beads to a magnet for 3 minutes and discarding the supernatant. This step was repeated three times. The peptide-coated beads could then be stored in the refrigerator at $4^{\circ} \mathrm{C}$ until ready to use.

Adsorption of rituximab from serum:

One hundred $\mu \mathrm{L}$ of the bead suspension were added to a $12 \times 75$ polystyrene tube. The peptide-coated beads were resuspended in $2.5 \mathrm{~mL}$ of BSA solution then adhered to a magnet for 3 minutes before decanting eliminated the supernatant. Normal human serum (NHS) containing rituximab or appropriate controls were then added to the beads and incubated for 15 minutes on a rocker at room temperature. The beads were removed by adherence to a magnet for 3 minutes. The serum was retained and further adsorbed as needed by repeating the above steps until no presence of rituximab was noted. The final adsorbed serum was now available for routine crossmatching techniques such as flow cytometry, or cytotoxicity assays.

Flow cytometric crossmatch:

Donor mononuclear cells were isolated by density gradient centrifugation. 250,000 isolated cells were incubated with purified anti-CD20 antibody (Becton Dickinson, San Jose, CA) for 10 minutes followed by $25 \mu \mathrm{L}$ of test (adsorbed) serum with incubation on ice. The cells were washed with BSA solution containing sodium azide. Binding of human IgG was detected with fluoresceinated (FITC) goat $\mathrm{F}(\mathrm{ab})_{2}$ anti-human IgG antibody (Jackson ImmunoResearch, West Grove, PA). The presence of CD19 or CD20 was detected with antibodies coupled to phycoerythrin (PE) (Becton Dickinson, San Jose, CA). Samples were analyzed by an Epics XL flow cytometer. Results are expressed as median fluorescence values (MFI) for green fluorescence (FITC) and red fluorescence (PE) and percent of positive cells.

\section{Results:}

A representative experiment is shown in figures 1 and 2. MFI of $\log$ green and red fluorescence and percent positive cells was compared for sera before and after treatment 
and with or without rituximab. MFI green fluorescence was 0.280 for NHS, 4.44 for NHS containing $10 \mu \mathrm{g} / \mathrm{mL}$ of rituximab before adsorption and 1.32 after 5 treatments. This indicated that less interfering rituximab was bound to the CD20 surface antigen expressed on $\mathrm{B}$ cells identified by binding of the goat anti-human IgG F(ab') ${ }_{2}$ FITC conjugated antibody. Further analysis showed that percent positive PE fluorescence measuring directly conjugated anti-human CD20 PE binding to the cells was 56.7 in NHS without rituximab, 0.84 for NHS containing $10 \mu \mathrm{g} / \mathrm{mL}$ of rituximab before adsorption, and 56.7 after 5 adsorptions. MFI of PE-conjugated anti-human CD20PE was 32.9 for NHS without rituximab, 0.903 for NHS containing $10 \mu \mathrm{g} / \mathrm{mL}$ of rituximab before adsorption, and 18.6 after 5 treatments. This indicated that rituximab interference had been reduced. Alloantibody binding was not affected by adsorption. With a pooled HLA-positive sera MFI for green fluorescence was 4.83 before and 4.11 after 5 treatments. No change was seen in CD19 with binding MFI red fluorescence was $4.26(75.2 \%+)$ before and 4.53 $(76.2 \%+)$ after 5 treatments. Complement dependent cytotoxicity (CDC) testing using these sera and cells from the same donor showed negative results for both $\mathrm{T}$ cell and $\mathrm{B}$ cell crossmatches for NHS, and NHS containing $10 \mu \mathrm{g} / \mathrm{mL}$ of rituximab after 5 treatments. B cell crossmatches were positive by CDC with NHS containing $10 \mu \mathrm{g} / \mathrm{mL}$ of rituximab and positive control. T cell crossmatches were positive for only positive control and negative for NHS containing $10 \mu \mathrm{g} / \mathrm{mL}$ of rituximab.

\section{Discussion:}

In the United States, the number of highly sensitized patients awaiting renal transplant have continued to rise with more 100,000 patients awaiting transplantation in 2014. with $14 \%$ having panel reactive antibody $>80 \%$. (5). Whether a patient has HLA antibodies to a given donor is commonly determined by a combination of solid phase detection assays and cell based crossmatch testing in the histocompatibility laboratory. Pretransaplant presence of HLA donor specific antibodies (DSA) is typically a contraindication for kidney transplantation. It has been previously shown that rituximab interferes with cell based crossmatch techniques such as the CDC assay and flow cytometric B cell crossmatching. Levels of rituximab have been shown to be present at 6 months with a single dose of $375 / \mathrm{m}^{2}$. (6.) Several methods have been described to remove rituximab 
from patient sera. The method we currently describe is an effective and more efficient way to remove rituximab from sera/plasma by allowing CD20 antigen beads to be prepared in bulk, validated and stored until required for use. There in minimal additional time required at the time of clinical testing whether before or after transplantation. This would facilitate the crossmatch testing of those patients who had received rituximab for clinical indications.

Pronase is proteolytic enzyme used frequently in histocompatibility laboratories at low doses to remove fc-like receptors from the cell surface of donor cells. While high doses of pronase effectively cleaves the CD20 that is also in the Fc receptor super family, from the surface of B cells, it is a harsh treatment. A number of cells are lost during this treatment. Loss of scarce donor cells could be a problem when crossmatching multiple donors or in post-transplant monitoring. As well, some investigators have shown that pronase may be inadequate for preparing cells for crossmatching with patients receiving rituximab (7).

Removing the interfering rituximab from the serum is another effective approach to this problem. However, there are currently no commercially available reagents that address this issue. While effective, the method described in Bearden et al. (3) requires multiple steps and may be considered impractical in the clinical situation.

We suggest that the method described in this paper is a viable option to eliminate the complications of interpreting crossmatch results in those patients who have received rituximab therapy. This method may be modified for use of elimination of other interfering chimeric antibodies as many more of these products are being used clinically.

\section{References:}

1. Pescovitz, MD. Rituximab, an anti-CD20 monoclonal antibody: Minireview: History and mechanisms of action. Am. J. Transplantation. 2006; 6 (5): 859-866. 
2. Bearden, CM, Agarwal, A, Book, BK, Sidner, RA, Gebel, HM, Bray, RA, and Pescovitz, MD. Pronase treatment facilitates alloantibody flow cytometric crossmatching in the presence of rituximab. Hum. Immunol. 2004; 65: 803-809.

3. Bearden, CM, Book, BK, Sidner, RA, Pescovitz, MD. Removal of therapeutic anti-lymphocyte antibodies from human sera prior to anti-human leukocyte antibody testing. J. Immonol. Methods. 2005; 300: 192-199.

4. Du, Jiamu, Wang, Hao, Zhong, Chen, Peng, Baozhen, Zhang, Li, Bohua, Huo, Sheng, Guo, Yajun and Jianping, Ding. Structural basis for recognition of CD20 by therapeutic antibody rituximab. J. of Biological Chemistry. 2007(282):1507315080 .

5. Mujtaba, MA, Komocsar, WJ, Nantz, E, Samaniego, MD, Henson, SL, Hague, JA, Lobashevsky, AJ, Higgins, N, Book, BK, Anderson, MD, Pescovitz, MD, Taber, TE. Effect of B-cell activating factor (BAFF) inhibition (LY2127399) tabalumab on highly sensitized patients with end stage kidney disease awaiting transplantation. American J of Transplantation. 2016; 16(4); 1266-1275.

6. Vierra CA, Agarwal A, Book B, et al. Rituximab for reduction of anti-HLA antibodies in patients awaiting renal transplantation: single dose safety and tolerability study. Transplantation. Feb 27, 2004;77(4):542-548.

7. Hetrick, SJ, Schillinger, KP, Zachary, AA, Jackson, AM. Impact of pronase on flow cytometric outcome. Hum. Immunol. 2011; 74(4): 330-336. 
Figure 1. Flow cytometric crossmatch histogram between donor spleen cells and normal human serum (in blue), normal human serum containing $10 \mu \mathrm{g} / \mathrm{mL}$ of rituximab (in green), and after 5 depletion treatments described herein (in orange), and a positive control containing alloantibody, but no rituximab (in red). The presence of rituximab's Fc portion gave a prominent positive peak comparable to alloantibody positive control (in red), a result of the recognition of FITC labeled antihuman IgG1. The reduced rituximab in the adsorbed serum was indicated by a smaller number of fluorescence labeled cells, recognized by FITC labeled anti-human IgG1. The peak positive channel shifted to the left of the histogram, and the intensity decreased indicating that after adsorption, less rituximab was bound to the cells to be identified by binding of the goat anti-human $\operatorname{IgG~F(ab')}{ }_{2}$ FITC conjugated antibody. 
Figure 2. Flow cytometric cross match histogram between donor spleen cells and normal human serum containing no rituximab (in red), normal human serum containing $10 \mu \mathrm{g}$ $/ \mathrm{mL}$ of rituximab (in green), and normal human serum containing $10 \mu \mathrm{g} / \mathrm{mL}$ of rituximab after 5 depletion treatments described herein (in orange), and a positive control containing alloantibody, but no rituximab (in blue). The fluorescent antibody used was phycoerytherin (PE) labeled anti-human CD20. In the absence of rituximab to compete with fluorescence anti-CD20, a positive binding of PE labeled anti-CD20 was detected in the donor cells, indicated by the positive peak in the histogram. The reduced peak with serum containing rituximab showed blocking of the CD20 antigen by rituximab. After 5 depletion treatments, reduced rituximab in the serum was indicated by a positive binding of PE labeled anti-CD20 comparable to the normal human serum. fluorescence labeled cells detected by PE anti-human CD20. The CD20 antigen detection was not effected by positive alloantibody. 


\section{Anti-Human IgG FITC}

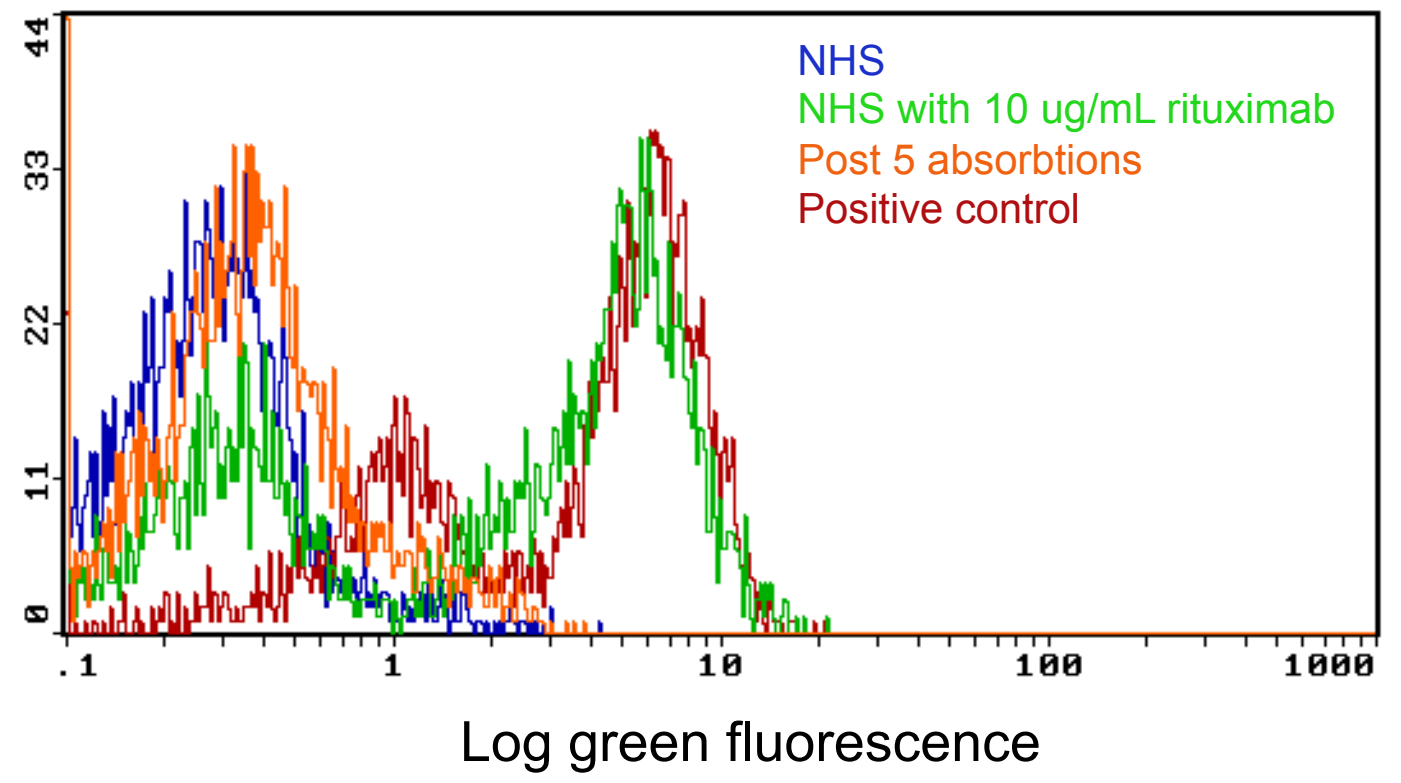




\section{Anti-CD20 PE}

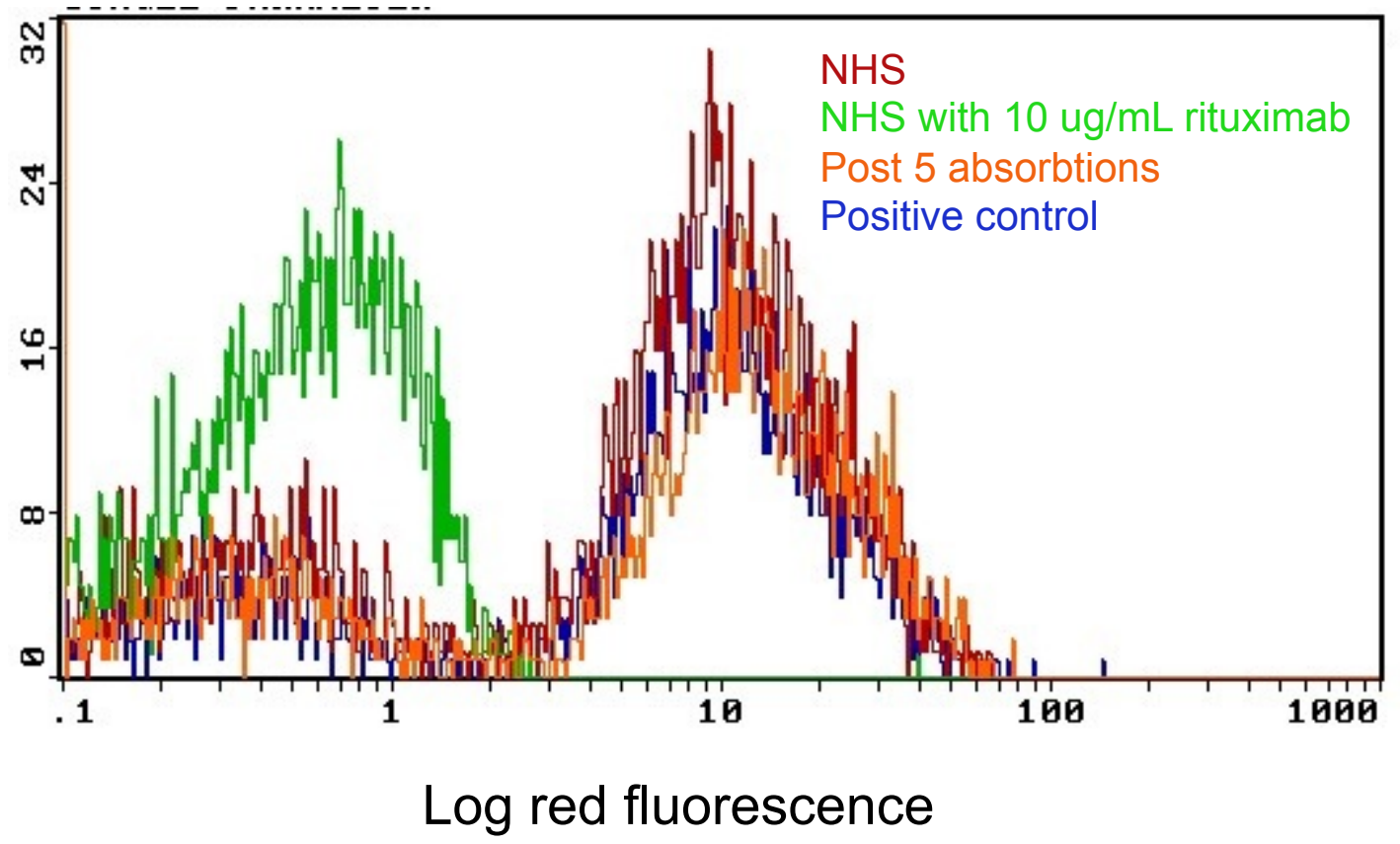

